\title{
Localizing Epileptogenic Zone from High Density EEG Data Using Machine Learning
}

\author{
https://doi.org/10.3991/ijoe.v17i06.18653 \\ Sehresh Khan, Aunsia Khan $\left({ }^{凶}\right)$ \\ National University of Modern Languages, Rawalpindi, Pakistan \\ aunsia.khan@numl.edu.pk \\ Nazia Hameed \\ University of Nottingham, Nottingham, UK \\ Muhammad Aleem Taufiq \\ University of Applied Sciences, Fachhochschule Fulda, Germany \\ Saba Riaz \\ Shaheed Zulfikar Ali Bhutto Institute of Science and Technology, \\ Karachi, Pakistan
}

\begin{abstract}
Drug-resistant focal epilepsy is the failure of antiepileptic drugs schedule to obtain epileptic free brain activities. In human brain cerebrum or cerebral hemispheres are the most commonly involved brain regions in epilepsy. In case of antiepileptic drugs failure, surgical treatment is the best cure possible for which correct localization of epileptogenic region is a challenging task for neurologists as well as for computer scientist for automatic localization. This research work's aim is to explore the functional activities of all brain regions in drug-resistant focal epileptic patients and achieve high accuracy for the classification of epileptogenic region (ER) with the high-density electroencephalographic (hdEEG) data. The proposed system includes frequency analysis for feature extractions followed by individual subject's registration of hdEEG signals with anatomical brain images for most precise localization of ER possible. The datasets attained from feature extraction process are then preprocessed for class imbalanced and then evaluated using different machine learning domains including the techniques under Bayesian networks, Lazy networks, Meta techniques, Rule based systems and Tree structured algorithms. Considering human brain both as stationary object as well as dynamic object, frequency-based and time frequency-based features are considered in 12 subjects respectively. Through this novel approach $99.70 \%$ accuracy is achieved to classify ER from healthy regions using KSTAR and IBK algorithm and $91.60 \%$ accuracy has been achieved to classify generator from propagator regions by applying IBK algorithm.
\end{abstract}

Keywords - Drug-resistant focal epilepsy, epileptogenic zone localization, computer-aided diagnosis, machine learning, epilepsy regions classification, high density EE 


\section{Introduction}

Epilepsy is complicated brain disease, which causes unpredictable functional activities of brain. These interruptions of normal brain functions are known as epileptic seizures. Epileptic patients face multiple frequencies of seizures, which are unprovoked. In some cases, this disease is cured by medicines but in some cases antiepileptic drugs do not work. In case of antiepileptic drugs failure, the main problem in the domain of epilepsy treatment is the identification of epileptic zone in the brain for a correct analysis and treatment. For such analysis, Brain of the patients are observed through multiple imaging techniques including magnetic resonance imaging (MRI), Diffusion spectrum magnetic resonance imaging (DSI), Diffusion Tensor Imaging (DTI) and many more. Diseases such as Epilepsy damage the brain cells and effect patient's daily life. Unlike other structural damages of the brain, identification of the brain zones generating or propagating the unhealthy signals (causing epilepsy) is much difficult. The abnormal functional and effective connectivity between different regions of the brain can be analyzed through fMRI, EEG, MEG and many other functional imaging techniques. Moreover, surgical procedures are mostly followed which are costly and even more challenging [1]. Therefore, especially in the epileptic patients who are drug resistant, it is important to identify correct epileptic region, before proceeding towards surgical evaluation.

Detection of epileptogenic zone through conventional scalp EEG system does not provide promising results especially for the frontal lobe epilepsy patients because of the fast cortical spread and artefacts caused by muscle movements [2]-[4]. In case of negative-MRI, mostly patients recommended and went through by intracranial EEG (iEEG). To get succeeded in the iEEG process with optimal number of intracranial electrodes, the most challenging and vital part is the correct identification of the epileptogenic region [5], [6]. However, high-density EEG increases the vision of whole neural network by covering most of the scalp area [7]. To overcome the risks involved in iEEG, hd-EEG played an important role to understand the neural network and the effective connectivity, abnormal electrical activities, spikes, seizures and clinical manifestations in different cortical and sub-cortical brain regions [8], [9]. The visual study of hd-EEG in focal epilepsy patients can provide a good improvement in localization of epileptogenic zone [9] but the problem with visual analysis is the requirement of expert's review which is not optimal automated solution.

In new era of artificial intelligence and machine learning, promising results are observed in current literature to analyze neural networks. Many studies have been carried out for the application of Machine learning on seizure detection and epileptic localization, however, ignoring the challenges while working on the complex datasets of neurological disorder. The most common classifiers for instance SVM [10, 11, 12], K-NN [13, 14], CNN [15-17], DT [18] and DF [19] has been applied for seizure detection, and localization using limited databases [20, 21]. The classifiers like K-NN is one of oldest classifiers that uses a simple logic to determine the output on the basis of k-nearest neighbors from feature set [22]. However, the main problem with k-NN is the majority voting especially when the data is skewed resulting into biased results for most frequent data sample. However, SVM is a binary classifier that make binary 
decisions. The problem with SVM is, it cannot make decision in case of nonlinear function without a kernel. The ADTree on the other hand creates a decision tree modified by boosting and is applied to two class problems. IB1, IBK and Kstar are simple instance-based learners that predicts the target class. RIDOR (Ripple down rule learner), on other hand, is a rule-based classifier that can classify data of various kind. In contrast, this research has been focused on using Bayes Network, Naïve Bayes [23], RBFNetwork [24], IB1, IBK, Kstar, Ridor, ADTree [25] classification techniques over High density complex EEG data. Due to the limitations and applicability of different classifiers, only these set of classifiers has been selected for the final experimentation purpose. To work with machine learning techniques the vital part is features selection. Features can be selected through channel based deep learning, image processing, signal processing or by using any statistical implications [26], [27]. For abnormal zone localization, EEG is analyzed per individual channels, with respect to individual frequency band. Moreover, classification techniques were used with all possible combinations and folds of EEG based plus relevant clinical features of the patients [28].

In this research work we presented a region-based analysis approach for the classification of epileptic brain regions from the healthy brain regions. For this purpose, high density EEG data is mainly considered to analyze the patients with focal epilepsy who were suggested for surgical treatment after the failure of antiepileptic drugs. For a better registration of EEG frequency signals on brain regions, each patient's personal anatomical images (MRI) are considered and registered with the Harvard-Oxford atlas (HOA) atlas. Considering the feature extraction, the most vital part of this approach, Fourier and Wavelet transform features are extracted for each brain region. As it is well known, frequency-based connectivity methods are based upon the spectral characteristics of the physiological signals and are able to differentiate causal interactions within specific frequency bands of interest [29],[30]. Instead, we hypothesize that the application of timevariant methods (e.g., ADTF) to EEG signals would allow capturing the dynamic evolution of the activity and characterizing the outgoing and ingoing information flow between different regions during an epileptic event.

This work is based on the estimation of functional connectivity in epilepsy allowing the identification of driving sources that are involved in inter-ictal activities as well as in the generation of seizure using EEG data. The region that is involved in the generation of the seizure is called generator while the propagator is the area which is normal healthy brain area without seizure. This study is divided into two groups of analysis, one to identify the epileptogenic regions from healthy regions and second the extraction of generator regions from propagators or healthy regions. Under both group of analysis, multiple experiments have been taken into account to cover all analytical aspects. The presented approach is implemented using different opensource software commonly available like MATLAB 2019, Brainstrom 3 and a comprehensive library of analysis tool known as FSL [31]. It is used for efficient analysis FMRI, MRI and DTI brain data.

The rest of the paper is organized as follows: The details of the proposed epileptogenic regions localization approach are explained in "Methodology" section. 
The Analyzed results of all the experiments are presented and discussed under the "Results" head. The approach presented in this paper with its outcome is then concluded in "Conclusions" section.

\section{Methodology}

\subsection{Sample selection}

Participants selected for this study are 12 patients diagnosed with drug resistant focal epilepsy including five male and seven female patients aged between 20 to 68 years. Patients were suffering from this disease from past 2 to 48 years. The average seizure frequency was 3.2 per day ranging between 1 per day to 30 per day.

All the patients were recommended for surgery and were admitted for the preassessments. To identify seizure semiotics, neurophysiological investigations including video-EEG, hdEEG [256-channel], arterial spin labelling (ASL), and electrical source imaging (ESI) has been performed. With the neurophysiological investigation techniques different advanced neuroimaging modalities were also analyzed to accurately localize epileptogenic zone. All the findings including neurophysiological and neuroimaging are presented in detail in [32], [33]. The modalities considered for this research are hdEEG, MRI, ESI and ASL with the major focus on hdEEG with actual MRI based atlas registration.

\subsection{Data acquisition}

High-density EEG with 256 channels (Electrical Geodesic, Inc., Eugene, OR) was performed by following international 10/20 system to evenly place electrodes on scalp surface. The hdEEG data were recorded with respect to a reference electrode (Cs). The sampling rate selected for recording was $250 \mathrm{~Hz}$. All the patients were guided to be in the rest position. The hdEEG recordings were further inspected and averaged with respect to peaks of spikes identified by expert neurophysiologist.

To study the dynamics of multiple brain regions, Harvard-Oxford atlas has been used. HOA divides the brain into 112 cortical and subcortical regions of interests (ROIs). To maintain every patient's individual peculiarities, HOA was registered on individual anatomical space perceived through each patient's T1-weighted MRI images as shown in figure 1(c). A source waveform for each ROI was calculated by averaging all the time series within the region represented in figure 1(e). The hdEEG data localization, registration of atlas with the patient's anatomical space and then finding of average time series for each ROI was evaluated by Brainstorm software [34]. Full process of Data acquisition through hdEEG including MRI brain registration, HOA atlas, Atlas registration, spike average calculated for each ROI and separate EEG spike of each region extracted of subject 1, are represented in figure $1(\mathrm{a}-\mathrm{e})$ respectively. 

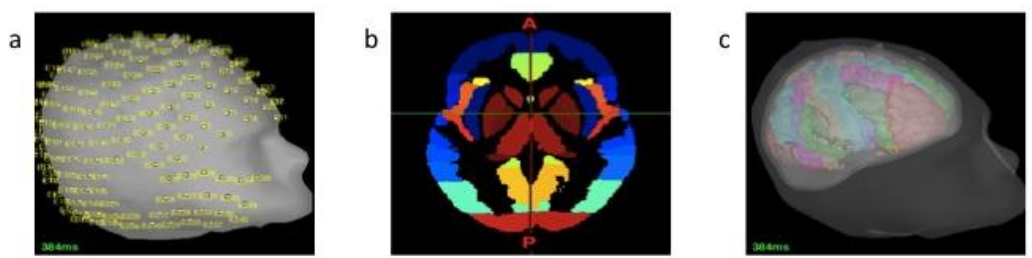

d

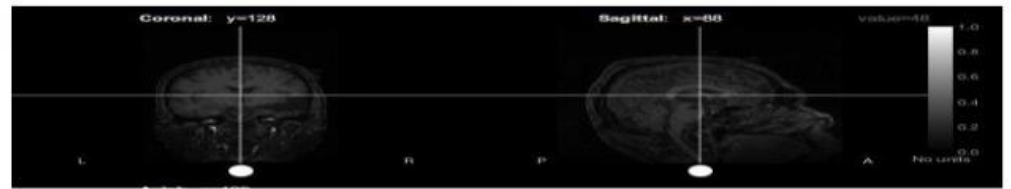

e

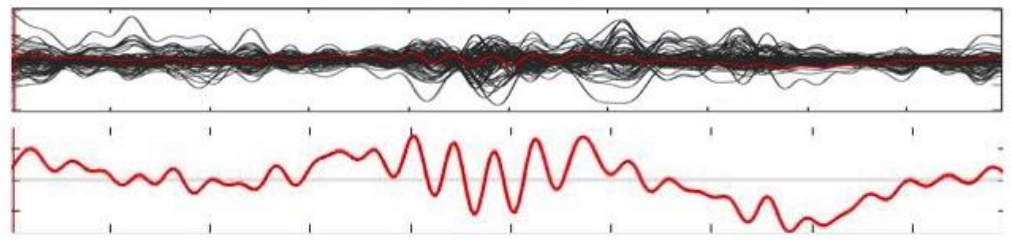

Fig. 1. Hd-EEG data mapping on subjects.01 individual anatomical image. a) Hd-EEG cap registration on subject's scalp surface. b) Harvard-Oxford atlas. c) HOA registered MRI based individual head surfaces. d) 3T anatomical MRI used for the registration of individual brain anatomy with the atlas regions and associated electrode spikes.

e) Average oscillation of all brain regions. f) Average spike of one ROI.

\subsection{Region of interests}

This study is divided into two groups of analysis as shown in Fig. 2; Group-I) Full brain analysis for the classification of healthy region (HR) and epileptogenic region (ER), Group-II) Brain's functionally active zone analysis for the prediction of Generator region (GR) among the Propagator Regions (PR).
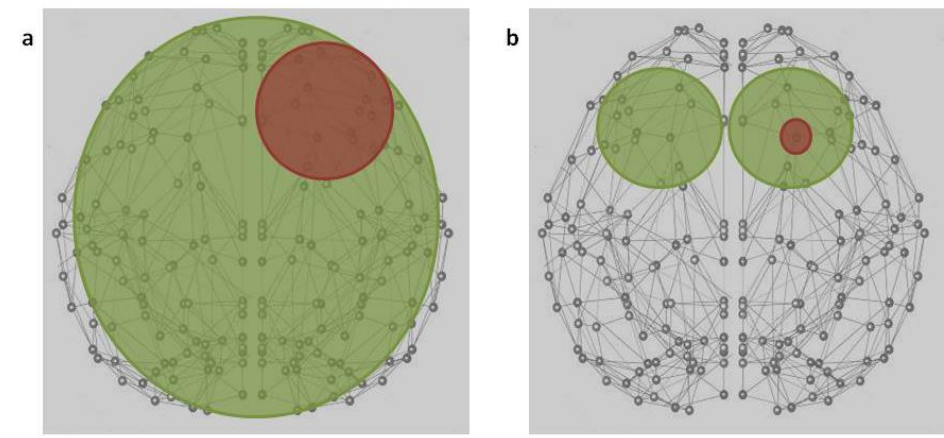

Epileptic Region(s) OHealthy Region(s)

Fig. 2. Two groups of Analysis. a) Full Brain Analysis.

b) Brain's functionally active zone analysis 
For first group of analysis common 104 regions of all patients are selected based on HOA. For all ROI hdEEG average time series data is considered as shown in figure $1(\mathrm{e}, \mathrm{f})$. For the analysis of second category, Five ROI based on the semeiotics from HOA of both Hemispheres for each patient is taken into consideration. These regions selection was based on previous research done by [8] which has shown the contribution of these regions independently as well as their effect in the form of a network. For each ROI, their Mean values of blood flow (CBF) and current density (CD) are also considered which were calculated previously for the research done by [32] and [33]. The most common brain regions involved in the functionally active zone analysis are listed in table 1.

Table 1. Common brain regions involved in the second category of analysis

\begin{tabular}{|c|l|l|c|c|}
\hline Sr. & Label & \multicolumn{1}{|c|}{ Name } & $\begin{array}{c}\text { No. of Voxels in } \\
\text { left hemisphere }\end{array}$ & $\begin{array}{c}\text { No. of Voxels in } \\
\text { Right hemisphere }\end{array}$ \\
\hline 1 & IFGo & Inferior frontal gyrus, pars opercularis & 236 & 200 \\
\hline 2 & IFGT & Inferior frontal gyrus, pars triangularis & 189 & 164 \\
\hline 3 & STGP & Superior temporal gyrus, posterior division & 119 & 118 \\
\hline 4 & MTGP & Middle temporal gyrus, posterior division & 403 & 390 \\
\hline 5 & MTGTo & Middle temporal gyrus, temporo-occipital part & 252 & 348 \\
\hline 6 & TP & Temporal pole & 707 & 691 \\
\hline 7 & STGa & Superior temporal gyrus, anterior division & 84 & 83 \\
\hline 8 & ITGa & Inferior temporal gyrus, anterior division & 103 & 103 \\
\hline 9 & FOrC & Frontal orbital cortex & 496 & 437 \\
\hline 10 & H & Hippocampus & 224 & 210 \\
\hline 11 & ITGto & Inferior temporal gyrus, temporo-occipital part & 211 & 126 \\
\hline 12 & MTGa & Middle temporal gyrus, anterior division & 128 & 172 \\
\hline 13 & ITGp & Inferior temporal gyrus, posterior division & 296 & 2377 \\
\hline 14 & FP & Frontal pole & 2045 & 123 \\
\hline 15 & FMC & Frontal medial cortex & 116 & 91 \\
\hline 16 & FOpC & Frontal operculum cortex & 102 & 86 \\
\hline 17 & TFCa & Temporal Fusiform Cortex, anterior division & 98 & 267 \\
\hline 18 & CoC & Central opercolar cortex & 278 & 140 \\
\hline 19 & PP & Planum temporale & 163 & \\
\hline & & & & \\
\hline
\end{tabular}

\subsection{Feature extraction}

For machine-learning data is the key to success however, the most difficult challenge is to apply it on medical images. Majority of medical imaging modalities are costly, time consuming and hectic especially for patients. For this study we tried to identify most optimal feature set using the above ROIs. We considered all possible features attained from any modality of all the subjects including demographic features as well as clinical. Some features are based on patients' demographic information like age, gender, epilepsy frequency etc and majority is with respect to each ROI obtained from functional imaging modalities. From hdEEG time series data, features are extracted with respect to different frequency bands as follows: Uper delta band (2-4 $\mathrm{Hz})$, theta band $(4-8 \mathrm{~Hz})$, alpha band $(8-12 \mathrm{~Hz})$, beta band $(12-30 \mathrm{~Hz})$, and gamma 
band $(30-50 \mathrm{~Hz})$. For each frequency band of each ROI, Fast Fourier Transform (FFT) and Continuous Wavelet Transform (CWT) is calculated. The FFT is a feature to represent the average frequency response in each band of each ROI. Equation (1) represents the delta band average frequency response (similar to all other selected frequency bands) is calculated as follows:

$$
\Delta i=\frac{\sum_{k=f s \Delta i}^{f n \Delta i}\left|A_{k}\right|}{f n \Delta i-f s \Delta i}
$$

where the starting frequency and maximum frequency in delta band is $f s \Delta i$ and $f n \Delta i$ respectively in a ROI $i . A$ is the Fourier coefficient for the input delta time series. Same method is applied for all band frequency responses calculation for all regions. After considering human brain as stationary system by calculating FFT feature considering brain as non-stationary dynamic system Continuous Wavelet Transform (CWT) features have been calculated. Continuous Wavelet Transform for Delta band $(\mathrm{CWT} \Delta)$ calculation is represented in equation (2) as follows:

$$
\Delta i=\frac{\sum_{l=t s \Delta i}^{t n \Delta i} \quad \sum_{k=f s \Delta i}^{f n \Delta i} \quad\left|X_{l, k}\right|}{(t n \Delta-t s \Delta)(f n \Delta i-f s \Delta i)}
$$

Where for the $i$ th ROI, the starting frequency and maximum frequency in delta band is $f s \Delta i$ and $f n \Delta i$ respectively where as $t s \Delta$ and $t n \Delta$ is the starting and ending time of the delta time series. The Current Density (CD) and Cerebral Blood Flow $(\mathrm{CBF})$ are also considered as clinical feature for brain active zone analysis. Current Density is calculated from ESI modality, whereas CBF is calculated from ASL modality by performing the equation (3) defined by [32] in detail.

$$
C B F=\frac{\Delta M}{2 \alpha M_{O b} T I_{1} e \frac{-\left(T I_{2}+(n-1) \text { slice } \text { time }\right)}{T_{1 b}}}
$$

where $\Delta M$ is the difference signal, $M_{o b}$ is the equilibrium magnetization of blood estimated from the calibration scan, $T I_{1}$ and $T I_{2}$ are the sequence time parameters, $\mathrm{n}$ is the number of a given slice, slice $_{\text {time }}$ is the time taken to acquire each slice ( 40 $\mathrm{ms}), T_{1 b}$ is the longitudinal relaxation time of blood (1664 ms at $\left.3 \mathrm{~T}\right)$, and $\alpha$ is the inversion efficiency ( 0.95 for pulsed ASL) [32]. As a demographic feature, age, years since beginning of the epilepsy, sex and seizure frequency per day of all the patients are considered in features set. All the ROI are then carefully labelled as HR or ER, to apply machine-learning techniques. In total 16 features were gathered for each participation.

\subsection{Classification analysis}

After finalizing all the calculations of feature data set, different type of analysis has been made to assess the role of these features to localize the epileptogenic zone.

Full brain analysis (Group-I): For localizing the epileptogenic region, using machine-learning techniques, representations of both classes are equally important therefore all 104 healthy and unhealthy ROIs are considered to train the model. For 
each ROI all 14 features including FFT and CWT features are considered. Under this group of analysis three types of experiments has taken place. 1) Considering only 1 region as epileptogenic ROI (ER) whereas all other are considered as healthy regions, 2) Considering most active 10 regions from both hemispheres as unhealthy participation in brain activity while others as healthy ROI and 3) Considering most active 5 regions belong to only one of the hemisphere identified as the epileptic hemisphere, as ER. The types of experiment under Group-I are presented in Fig.3. These all experiments are defined to assess the effectiveness of this research method for the most accurate localization possible using hdEEG and demographic data.

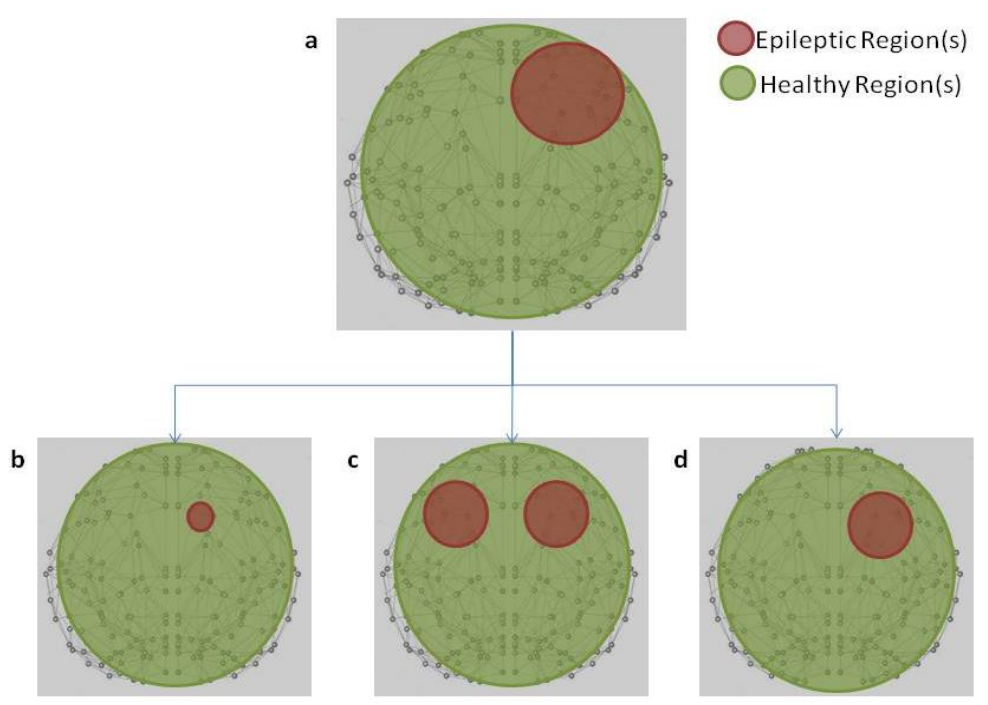

Fig. 3. a) Experiments under Full Brain Analysis. b) Exp-1: 1 ER.

c) Exp-2: 5 ER of both hemispheres. d) Exp-3: 5 ER of one hemisphere.

Functionally active brain zone analysis (Group-II): Epileptic region localizing problem is not only localization of brain zone but also the exact region localization under the effected zone to further proceed for the surgical treatment. From all the subjects 10 regions are considered which were actively participating in brain functionality while performing hdEEG in both hemispheres. Only 1 ROI is consider as ER and the rest of 9 ROIs are considered as HR. Along with hdEEG features, ESI and ASL features are also considered to more precisely classify generator and propagator regions among the functionally active brain zone. These regions selection and labeling is based on earlier findings in $[8,32,33]$.

For this group of analysis, 16 features of all ROI of each subject including FFT, CWT, CBF and CD are considered. To analyze the contribution of CBF and CD features in correct classification of generator as ER and propagators as HR two types of experiment has taken place under this group of analysis. 1) With CD and CBF features, 2) Without CD and CBF features. 
Classification methods: After the construction of three full brain analysis and two active brain analysis datasets, different classification techniques with 10-fold crossvalidation have been applied. These experiments are done by Weka version 3.8.3 [35]. For finding the most suitable classifier for the considered domain and under the complexity of features set, more than 25 classifiers from different classification domains like bayes, Lazy networks, Meta, Rule based and Tree structured were considered. Among them 9 classifiers i.e. Bayes Network, NaiveBayes [24], RBFNetwork [25], IB1, IBK, Kstar, Ridor, ADTree [26] and Ordinal CC are considered for comparative analysis because of their outperformance than others.

Evaluation and visualization: Using the above methodology, we have taken the five different experimental datasets including 3 from Group-I and 2 from Group-II as explained above. Initially, the data is having less numbers of effected regions in comparison to unaffected regions hence, creating class imbalance. It leads to the biased results as the classifier always focus on the majority class and produce high accuracies for it while producing the poor results for minority class. Therefore, class imbalance issue is being solved in this research using SMOTE [36].

For evaluating the classification result, 10 -fold cross-validation has been used. To evaluate the performance of the classification models following measures have been calculated

1. Accuracy

2. Recall

3. F-Measure

\section{$3 \quad$ Results}

While comparing different type of classification methodologies and techniques, time complexity measurement and space complexity measurement are two key factors to be analyzed.

The classification results for each experiment under both groups of analysis can be seen in Table 2 and Table 3. It is noticeable that, for all experiments of both categories, the classification algorithms produce high classification accuracy with high percentage of recall and F-measure. However, it can be noted that the highest classification accuracy is achieved by the Kstar Classifier i.e. 99.79\% accuracy with 99.6\% Recall and 99.8\% F-Measure in experiment 1 of Group-I where the affected area as ER is localized to only one region. 
Table 2. Results; Classifiers Accuracy, Recall and F-measure percentage achieved in all experiments.

\begin{tabular}{|c|c|c|c|c|c|c|c|c|c|}
\hline \multirow{3}{*}{$\begin{array}{c}\text { ML. } \\
\text { Algorithms }\end{array}$} & \multicolumn{9}{|c|}{ Group-I: Full Brain Analysis } \\
\hline & \multicolumn{3}{|c|}{ Exp1 } & \multicolumn{3}{|c|}{$\operatorname{Exp2}$} & \multicolumn{3}{|c|}{$\operatorname{Exp3}$} \\
\hline & Accuracy & Recall & $\begin{array}{c}F- \\
\text { Measure }\end{array}$ & Accuracy & Recall & $\begin{array}{c}F- \\
\text { Measure }\end{array}$ & Accuracy & Recall & $\begin{array}{c}F- \\
\text { Measure }\end{array}$ \\
\hline BayesNet & 99.3 & 99.6 & 99.3 & 92.2 & 96.7 & 92.6 & 95.4 & 98.3 & 95.5 \\
\hline NaiveBayes & 99.3 & 99.7 & 99.3 & 91.9 & 96.7 & 92.3 & 95.4 & 98.3 & 95.5 \\
\hline RBFNetwork & 99.4 & 99.7 & 99.4 & 84.4 & 97.2 & 86.2 & 79.3 & 98.5 & 82.6 \\
\hline IB1 & 99.5 & 99.6 & 99.6 & 77.3 & 96.4 & 81 & 84.4 & 98.1 & 86.3 \\
\hline IBK & 99.7 & 99.6 & 99.7 & 83.5 & 95.3 & 85.2 & 80.6 & 98.1 & 83.5 \\
\hline Kstar & 99.7 & 99.6 & 99.8 & 81.1 & 95.8 & 83.6 & 80.3 & 98.2 & 83.3 \\
\hline Ridor & 99.5 & 99.2 & 99.5 & 95.6 & 91.2 & 95.4 & 97.4 & 95.2 & 97.4 \\
\hline ADTree & 99.5 & 99.5 & 99.5 & 86.7 & 76.1 & 85.2 & 97.4 & 95.4 & 97.4 \\
\hline
\end{tabular}

Table 3. Results; Classifiers Accuracy, Recall and F-measure percentage achieved in all experiments.

\begin{tabular}{|l|c|c|c|c|c|c|}
\hline \multirow{2}{*}{ ML. Algorithms } & \multicolumn{6}{|c|}{ Group-II: Active Brain Analysis } \\
\cline { 2 - 7 } & \multicolumn{3}{|c|}{ Exp2 } \\
\cline { 2 - 7 } & Accuracy & Recall & F-Measure & Accuracy & Recall & F-Measure \\
\hline BayesNet & 75.4 & 76.9 & 75.8 & 51.8 & 31.5 & 39.5 \\
\hline NaiveBayes & 70.8 & 83.3 & 74.1 & - & - & - \\
\hline RBFNetwork & 73.1 & 78.7 & 74.6 & 54.1 & 65.7 & 58.9 \\
\hline IB1 & 76.4 & 74.1 & 75.8 & 87 & 83.3 & 86.5 \\
\hline IBK & 76.4 & 66.7 & 73.8 & 91.6 & 83.3 & 90.9 \\
\hline Kstar & 79.1 & 75.9 & 78.5 & 82.8 & 65.7 & 79.3 \\
\hline Ridor & 77.7 & 78.7 & 78 & 84.7 & 73.1 & 82.7 \\
\hline ADTree & 81.4 & 82.4 & 81.7 & 89.8 & 80.6 & 88.8 \\
\hline
\end{tabular}

For the cross validation of the proposed system of epileptic region localization using machine learning the average outcomes are also analyzed. The average accuracy in all experiments lied between the ranges of $69.34 \%$ to $99.54 \%$ as represented in table 4. By considering all the measures i.e. Accuracy, Recall and F-Measure, by considering average of all the classifiers, experiment 1 of Group-I outperformed all other experiments in both groups.

Table 4. Average Accuracy, Recall and F-measure percentage in all experiments.

\begin{tabular}{|l|c|c|c|c|c|}
\hline \multirow{2}{*}{ Average Measures } & \multicolumn{3}{|c|}{ Group-I } & \multicolumn{2}{c|}{ Group-II } \\
\cline { 2 - 6 } & Exp.1 & Exp.2 & Exp.3 & Exp.1 & Exp.2 \\
\hline Accuracy & 99.52 & 86.62 & 89.49 & 76.39 & 69.34 \\
\hline Recall & 99.50 & 93.19 & 97.41 & 77.48 & 60.79 \\
\hline F-Measure & 99.51 & 87.66 & 90.72 & 76.67 & 67.18 \\
\hline
\end{tabular}

However, with Fourier and Wavelet transform features, density of current flow and blood flow are also considered for the classification of generator from propagators within epileptic brain zone which reflected decline of accuracy from $76.39 \%$ to 
$69.34 \%$ on average among multiple classifiers. There is no significance of CD and $\mathrm{CBF}$ features is observed because the majority of the areas considered were already identified by increasing CD and decreasing CBF values trend as compared to healthy controls by [16]. There is no such distinguishing factor of these values among the considered regions to classify genrator verses propgators in Experiment 2 of active brain analysis (Group-II). These features can be of massive participation if would be used for full brain analysis (Group-I) experiments to classify ER(s) from HR(s) which is not easily applicable because of clinical limitations.

On average the recall percentage lied between the ranges of $60.79 \%$ to $99.50 \%$ whereas the average F-measure lied between the ranges of $67.18 \%$ to $99.51 \%$. The highest rate of accuracy, recall and f-measure is achieved in experiment 1 as listed in table 3. For experiment 2 and 3 of Group-I analysis and experiment 1 of Group-II analysis, more recall percentage is achieved as compared to accuracy and F-measure whereas in experiment 1 of Group-I, same results are achieved for accuracy, recall and f-measure and in experiment 2 of Group-II, high accuracy is achieved as compared to recall and F-measure.

Fig. 4 represents all the measures calculated in all experiments for this research work as a spiral graph. As shown in the figure 3, experiment 1 of Group-I outperformed in all the measures and touched the upper bound near $100 \%$ achievement.
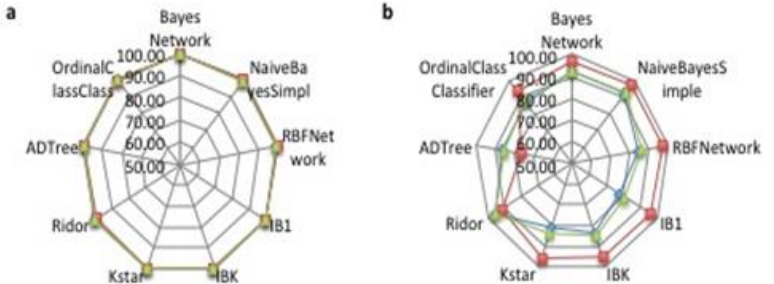

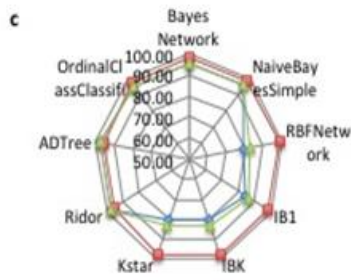

d

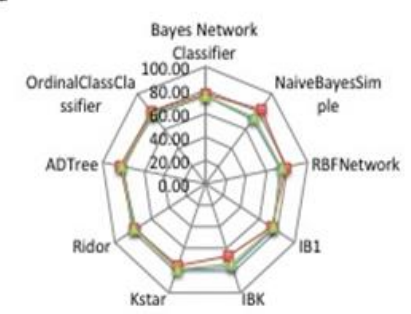

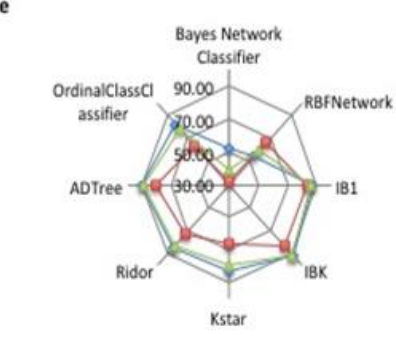

Fig. 4. Accuracy, Recall and F-measure comparison for different classifiers. a) Full brain analysis considering one ER. b) Full brain analysis considering ten ER(s) across both hemispheres. c) Full brain analysis considering five ER(s) of one hemisphere.

d) Active brain analysis including $\mathrm{CD}$ and $\mathrm{CBF}$ measures.

e) Active brain analysis without $\mathrm{CD}$ and $\mathrm{CBF}$ measures 


\section{Conclusion}

A novel approach for the localization of epileptic region is presented in this research to classify epileptogenic brain regions from healthy brain regions based on high density EEG data using different open source softwares like MATLAB 2019, Brainstrom 3 and FSL. This approach is based on rich features extracted through signal processing and classification using several machine learning algorithms. This research is majorly divided into two categories; 1) classification of epileptic region from healthy brain regions, 2) classification of generator region from the propagator regions of epileptic brain zone. The results of this research validated both approaches by achieving the classification accuracy up to $99.5 \%$ in first category by only considering features based on hdEEG epochs of 1 second each. Whereas the results of this research also validated the contribution of features like blood flow (CBF) and current density (CD) are extracted from other modalities such as ESI and ASL to classify generator regions from the propagator regions with the classification accuracy of $91.6 \%$.

To conclude, the approach adopted in this research for the localization problem of the drug resistant focal epileptic patients, high accuracy has been achieved using multiple machine learning algorithms. Fourier transform features and wavelet transform features are the major contribution in feature set for full brain classification into ER and HR. This research can be further explored for the more accurate classification of generator from the propagator regions by considering multiclass classifiers and features from other clinical biomarkers.

\section{$5 \quad$ References}

[1] H. Huppertz, E. Hof, J. Klisch, M. Wagner, C. Lücking and R. Kristeva-Feige, "Localization of Interictal Delta and Epileptiform EEG Activity Associated with Focal Epileptogenic Brain Lesions", NeuroImage, vol. 13, no. 1, pp. 15-28, 2001. https://doi.org/ 10.1006/nimg.2000.0680.

[2] R. D. Bautista, D. Spencer and S. Spencer, "EEG findings in frontal lobe epilepsies", Neurology, vol. 50, no. 6, pp. 1765-1771, 1998. https://doi.org/10.1212/wnl.50.6.1765.

[3] C. Catarino, C. Vollmar and S. Noachtar, "Paradoxical lateralization of non-invasive electroencephalographic ictal patterns in extra-temporal epilepsies", Epilepsy Research, vol. 99, no. 1-2, pp. 147-155, 2012. Available: https://doi.org/10.1016/j.eplepsyres.2011 .11 .002

[4] N. So, "Mesial Frontal Epilepsy", Epilepsia, vol. 39, no. 4, pp. S49-S61, 1998. Available: https://doi.org/10.1111/j.1528-1157.1998.tb05125.x

[5] T. Abuelem, D. Friedman, S. Agadi, A. Wilfong and D. Yoshor, "Interhemispheric Subdural Electrodes: Technique, Utility, and Safety", Operative Neurosurgery, vol. 73, no. 2, pp. ons253-ons260, 2013. Available: https://doi.org/10.1227/01.neu.0000430287.0855 2.83

[6] J. Van Gompel et al., "Intracranial Electroencephalography with Subdural Grid Electrodes", Neurosurgery, vol. 63, no. 3, pp. 498-506, 2008. Available: http://doi.org/10. 1227/01.neu.0000324996.37228.f8.

[7] C. Plummer, A. Harvey and M. Cook, "EEG source localization in focal epilepsy: Where are we now?", Epilepsia, vol. 49, no. 2, pp. 201-218, 2008. Available: https://doi.org/10. $\underline{1111 / \mathrm{j} .1528-1167.2007 .01381 . x}$ 
[8] S. Storti, I. Galazzo, S. Khan, P. Manganotti and G. Menegaz, "Exploring the Epileptic Brain Network Using Time-Variant Effective Connectivity and Graph Theory", IEEE Journal of Biomedical and Health Informatics, vol. 21, no. 5, pp. 1411-1421, 2017. Available: https://doi.org/10.1109/jbhi.2016.2607802

[9] A. Feyissa et al., "High density scalp EEG in frontal lobe epilepsy", Epilepsy Research, vol. 129, pp. 157-161, 2017. Available: https://doi.org/10.1016/j.eplepsyres.2016.12.016

[10] Z. Zisheng and P. KK, "Seizure detection using wavelet decomposition of the prediction error signal from a single channel of intra-cranial EEG", in 36th annual international conference of the IEEE engineering in medicine and biology society., 2014. https://doi.org/ $10.1109 / \mathrm{embc} .2014 .6944610$

[11] V. Chavakula et al., "Automated quantification of spikes", Epilepsy \& Behavior, vol. 26, no. 2, pp. 143-152, 2013. Available: http://doi.org/10.1016/j.yebeh.2012.11.048.

[12] C. Cortes and V. Vapnik, "Support-vector networks", Machine Learning, vol. 20, no. 3, pp. 273-297, 1995. Available: http://doi.org/10.1007/bf00994018.

[13] S. Siuly, E. Kabir, H. Wang and Y. Zhang, "Exploring Sampling in the Detection of Multicategory EEG Signals", Computational and Mathematical Methods in Medicine, vol. 2015, pp. 1-12, 2015. Available: https://doi.org/10.1155/2015/576437

[14] H. Firpi, E. Goodman and J. Echauz, "On Prediction of Epileptic Seizures by Means of Genetic Programming Artificial Features", Annals of Biomedical Engineering, vol. 34, no. 3, pp. 515-529, 2006. Available: https://doi.org/10.1007/s10439-005-9039-7

[15] Y. Yuan, G. Xun, K. Jia and A. Zhang, "A multi-context learning approach for EEG epileptic seizure detection", BMC Systems Biology, vol. 12, no. 6, 2018. Available: https://doi.org/10.1186/s12918-018-0626-2

[16] M. Zhou et al., "Epileptic Seizure Detection Based on EEG Signals and CNN", Frontiers in Neuroinformatics, vol. 12, 2018. Available: http://doi.org/10.3389/fninf.2018.00095.

[17] A. Emami, N. Kunii, T. Matsuo, T. Shinozaki, K. Kawai and H. Takahashi, "Seizure detection by convolutional neural network-based analysis of scalp electroencephalography plot images", NeuroImage: Clinical, vol. 22, p. 101684, 2019. Available: https://doi.org/ 10.1016/j.nicl.2019.101684

[18] S. Salzberg, "C4.5: Programs for Machine Learning by J. Ross Quinlan. Morgan Kaufmann Publishers, Inc., 1993", Machine Learning, vol. 16, no. 3, pp. 235-240, 1994. Available: $10.1007 /$ bf00993309. https://doi.org/10.1007/bf00993309

[19] L. Breiman, "Random forests". Machine learning, vol. 1, no. 45, pp.5-32, 2001.

[20] B. Sharif and A. Jafari, "Prediction of epileptic seizures from EEG using analysis of ictal rules on Poincaré plane", Computer Methods and Programs in Biomedicine, vol. 145, pp. 11-22, 2017. Available: https://doi.org/10.1016/j.cmpb.2017.04.001

[21] C. Alexandre Teixeira et al., "Epileptic seizure predictors based on computational intelligence techniques: A comparative study with 278 patients", Computer Methods and Programs in Biomedicine, vol. 114, no. 3, pp. 324-336, 2014. Available:. https://doi.org/ 10.1016/j.cmpb.2014.02.007

[22] A. Khan and M. Usman, "Alzheimer's Disease Prediction Model Using Demographics and Categorical Data", International Journal of Online and Biomedical Engineering (iJOE), vol. 15, no. 15, p. 96, 2019. Available: https://doi.org/10.3991/ijoe.v15i15.11472

[23] Abbasi, B., \& Goldenholz, D. M. (2019). Machine learning applications in epilepsy. Epilepsia, 60(10), 2037-2047. https://doi.org/10.1111/epi.16333

[24] Satapathy, S. K., Dehuri, S., \& Jagadev, A. K. (2017). ABC optimized RBF network for classification of EEG signal for epileptic seizure identification. Egyptian Informatics Journal, 18(1), 55-66. https://doi.org/10.1016/j.eij.2016.05.001

[25] P. Kovacs, K. Samiee and M. Gabbouj, "On application of rational Discrete Short Time Fourier Transform in epileptic seizure classification," 2014 IEEE International Conference on Acoustics, Speech and Signal Processing (ICASSP), Florence, 2014, pp. 5839-5843. https://doi.org/10.1109/icassp.2014.6854723 
[26] P. Durongbhan et al., "A Dementia Classification Framework Using Frequency and TimeFrequency Features Based on EEG Signals", IEEE Transactions on Neural Systems and Rehabilitation Engineering, vol. 27, no. 5, pp. 826-835, 2019. Available: https://doi.org/10. $1109 /$ tnsre.2019.2909100

[27] T. Alotaiby, S. Alshebeili, T. Alshawi, I. Ahmad and F. Abd El-Samie, "EEG seizure detection and prediction algorithms: a survey", EURASIP Journal on Advances in Signal Processing, vol. 2014, no. 1, 2014. Available: https://doi.org/10.1186/1687-6180-2014-183

[28] P. Durongbhan et al., "A Dementia Classification Framework Using Frequency and TimeFrequency Features Based on EEG Signals", IEEE Transactions on Neural Systems and Rehabilitation Engineering, vol. 27, no. 5, pp. 826-835, 2019. Available: https://doi.org/10. $1109 /$ tnsre.2019.2909100

[29] B. He, Y. Dai, L. Astolfi, F. Babiloni, H. Yuan, and L. Yang, "eConnectome: A MATLAB toolbox for mapping and imaging of brain functional connectivity," J. Neurosci. Methods, vol. 195, pp. 261-269, Feb 2011. https://doi.org/10.1016/j.jneumeth.2010.11.015

[30] K. Ayodele, W. Ikezogwo and A. Osuntuyi, "Empirical Characterization of the Temporal Dynamics of EEG Spectral Components", International Journal of Online and Biomedical Engineering (iJOE), vol. 16, no. 15, p. 80, 2020. Available: https://doi.org/10.3991/ ijoe.v16i15.16663

[31] M. Jenkinson, C.F. Beckmann, T.E. Behrens, M.W. Woolrich, S.M. Smith. FSL. NeuroImage, 62:782-90, 2012 https://doi.org/10.1016/j.neuroimage.2011.09.015

[32] S. Storti et al., "Combining ESI, ASL and PET for quantitative assessment of drugresistant focal epilepsy", NeuroImage, vol. 102, pp. 49-59, 2014. Available: https://doi.org/ 10.1016/j.neuroimage.2013.06.028

[33] I. Boscolo Galazzo et al., "Patient-Specific Detection of Cerebral Blood Flow Alterations as Assessed by Arterial Spin Labeling in Drug-Resistant Epileptic Patients", PLOS ONE, vol. 10, no. 5, p. e0123975, 2015. Available: https://doi.org/10.1371/journal.pone.0123975

[34] F. Tadel, S. Baillet, J. Mosher, D. Pantazis and R. Leahy, "Brainstorm: A User-Friendly Application for MEG/EEG Analysis", Computational Intelligence and Neuroscience, vol. 2011, pp. 1-13, 2011. Available: https://doi.org/10.1155/2011/879716

[35] S. Srivastava, "Weka: A Tool for Data preprocessing, Classification, Ensemble, Clustering and Association Rule Mining", International Journal of Computer Applications, vol. 88, no. 10, pp. 26-29, 2014. Available: https://doi.org/10.5120/15389-3809

[36] N. Chawla, K. Bowyer, L. Hall and W. Kegelmeyer, "SMOTE: Synthetic Minority Oversampling Technique", Journal of Artificial Intelligence Research, vol. 16, pp. 321-357, 2002. Available: 10.1613/jair.953. https://doi.org/10.1613/jair.953

\section{Authors}

Sehresh Khan and Aunsia Khan works at National University of Modern Languages, Rawalpindi, Pakistan. Available on mail at aunsia.khan@numl.edu.pk

Nazia Hameed is with School of Computer Science, University of Nottingham, $\mathrm{UK}$

Muhammad Aleem Taufiq works at University of Applied Sciences, Hochschule Fulda, Germany

Saba Riaz is with Shaheed Zulfikar Ali Bhutto Institute of Science and Technology, Islamabad, Pakistan

Article submitted 2020-09-19. Resubmitted 2021-04-01. Final acceptance 2021-04-04. Final version published as submitted by the authors. 